\title{
Management of Electrical Energy of a Multi-source System Using FPGA Card
}

\author{
R. Alkama and A. Bouzeroura
}

\begin{abstract}
This work is a part of the application using a standalone hybrid power generation system from photovoltaic panels, batteries and generators. Storage management and distribution of energy to the receivers will optimize the power of the battery and the operating time of the generator. To enable this optimization we proposed to use a FPGA card which manages the power transfer to the receivers according to the required energy. It allows to make maximum use of photovoltaic energy and to minimize the amount of fuel source relief and the air pollution emissions.

The integration of FPGA systems in the management of energy sources is used to study and get a simulation model which can validate a solution before making the installation. The simulation of a battery power management can facilitate full simulation of the energy system.

Our purpose is to have an optimized management model of a hybrid system, obtained by a combination of several submodels of each source controlled separately.
\end{abstract}

Index Terms-Application VHDL, batteries, PV, generator, optimization.

\section{INTRODUCTION}

Electrical energy demand by populations and limited classic resources, make that renewable energy is becoming very interesting in several areas, providing a real economic and environmental solutions. Autonomous solar or wind systems can play a very important role. The amount of light captured by solar panels inevitably depends on weather conditions (ambient temperature, relative humidity, atmospheric pressure, wind speed and direction) [1].

Optimization of electrical power supply sources can be multi-accomplished in several ways [2], [3].

The application of VHDL is widely used following several methodologies, which allowed the different areas change very quickly, exploiting new technologies, handling specifications and functionality of systems according to market needs [4]-[6]. We present an example of application of VHDL for the optimized management of electrical energy from the batteries of our multi-source system.

We must simulate the energy management model of batteries storage for our hybrid system and validate the result before making the FPGA board.

\section{PURPOSE}

The idea is to develop a system for optimized power management for multi-source system with VHDL (VHSIC

Manuscript received February 1, 2016; revised May 28, 2016.

The authors are with the Laboratory of Electrical Engineering, University of Bejaia, Algeria (e-mail: rezak_alkama@yahoo.fr, ghanielm2006@yahoo.fr).
Hardware Description Language) and implemented on FPGA (Feed Programmable Gate Array) XILINX a capture card adaptable to all equipment types. The design of the VHDL code must remain generic and configurable by the user on the PC without reprogramming the FPGA.

The purpose of this project is to provide a flexible FPGA with a condition that each Input / Output can achieve the desired function with appropriate programming.

Therefore, it provides a program for each I / O initially.

\section{THE SPECIFICATION}

Solar and wind power modules to determine the energy that can provide production systems for weather data and technology. These are supplied to the optimization tool with the consumption data, the characteristics of the network, the storage device and UPS as well as their energy performance and cost functions. The optimization tool then gives us the dimensions of the various elements of the system and the optimal strategy of energy transfers. Another advantage of this mounting PV-wind - GE- battery - load in parallel is that the battery works as tension regulator to supply the load. The battery voltage requires parallel arrangement and thus stabilizes the voltage supplied to the load, which is an advantage.

Taking a part of this system is that the battery packs as an example of VHDL application. Battery management is done independently and is based on two basic criteria: protection against deep discharge and optimal management.

We adopt an on/off regulation which is represented by $0 / 1$ for battery management based on the battery voltage disconnects and reconnects according to four thresholds.

\section{A. Organization}

Fig. 1 shows the flow chart of battery management unit.

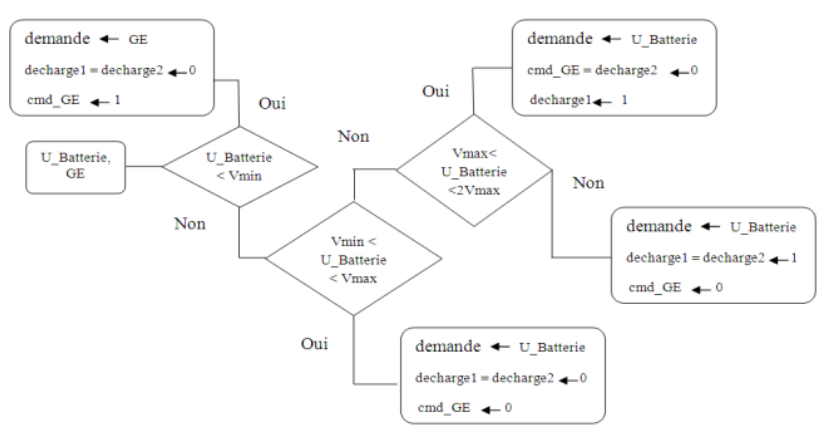

Fig. 1. Battery management unit.

Different thresholds are:

- V4: high cutoff threshold, English HVD for "High Voltage Disconnect" that corresponds to the threshold voltage of the battery when fully charged; then it stops 
charging: "Off."

- V3: high reconnection threshold: it allows to introduce a hysteresis to avoid pumping phenomena controller; charging does not resume until the battery voltage is lower than $\mathrm{V} 3$.

- V2: low threshold of reconnection: it introduces a second order to avoid the effects of pumping when the battery is in a state of discharge. When the battery reaches its minimum operating voltage (voltage fixed according to the desired depth of discharge) and recharging follows; reconnection of the load is possible only when the battery voltage is higher than V2.

- V1: low cutoff; when the battery voltage reaches the low cutoff, we consider it completely discharged, it is necessary to disconnect the load.

For the battery role in the optimization of multi-source for energy system, and the various interactions between the battery and the input / output system, we propose the diagram of Fig. 2.

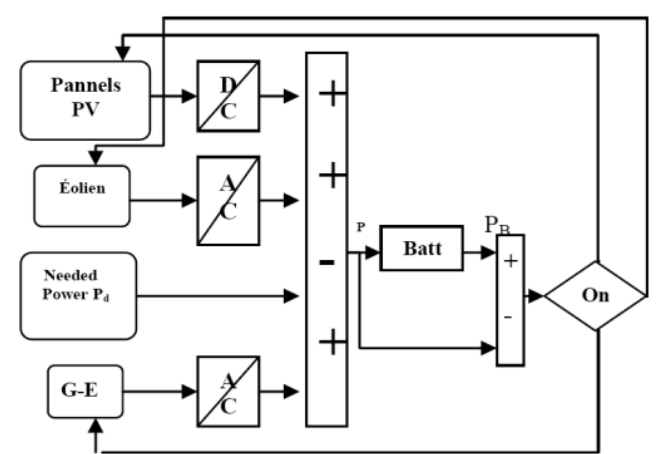

Fig. 2. Management of battery power to a multi-source system.

\section{B. Programming and Simulation}

In this step, we define the overall system design, namely inputs and outputs. The inputs are: the battery voltage "U_Batterie", the output voltage of the generator (GE), which are digital signals 32 bits, and outputs are: commands (ON / OFF) to bit (0/1) to "load 1", "load2 " and manipulating control ignition GE "cmd_GE" and the consumer demand "demand" is a 32-bit digital signal [5], [6], see Fig. 3.

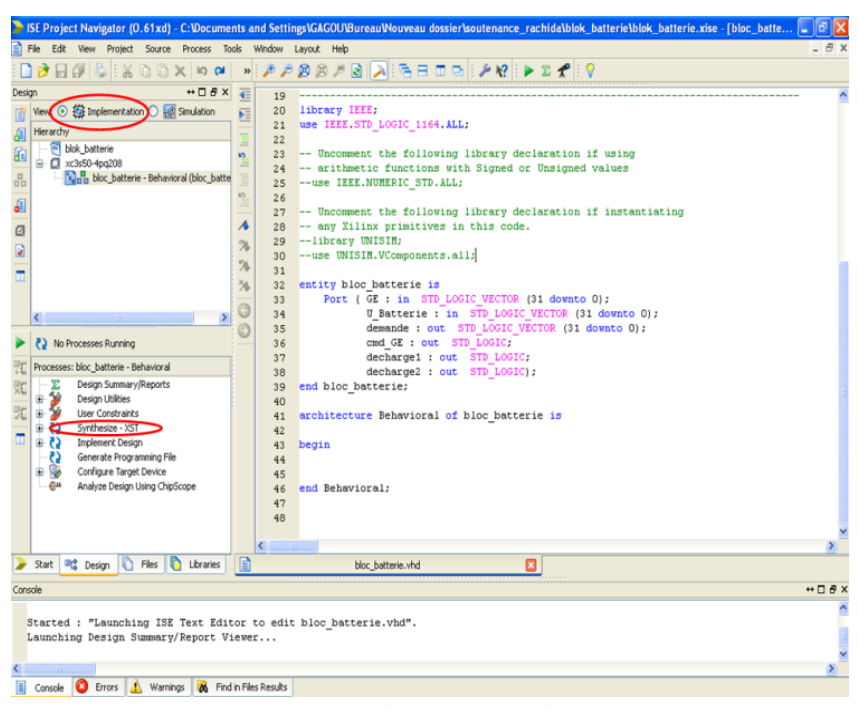

Fig. 3. Visualization of the resulting file battery pack.
Summary: Let us detail a little in the system to visualize the resulting circuit, according to Fig. 2.

Fig. 4 and Fig. 5 show a simplified schematic circuit design for battery power management, with three inputs and three outputs.

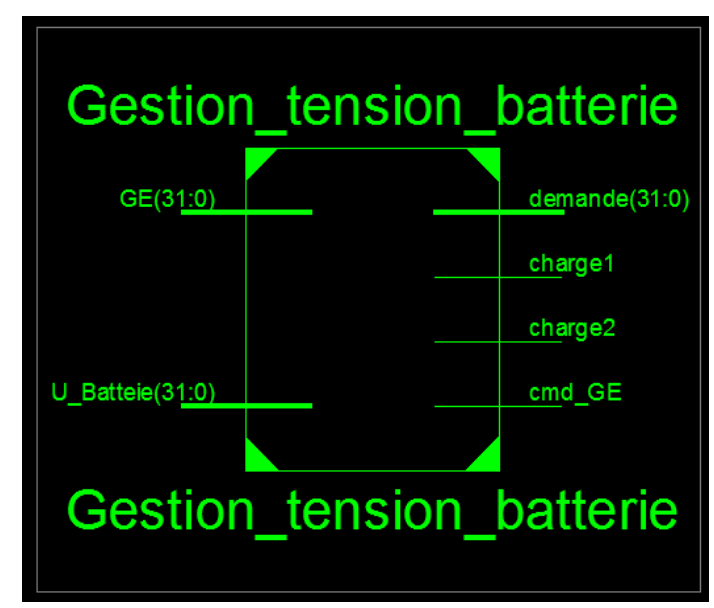

Fig. 4. Synthesis of model management battery pack.

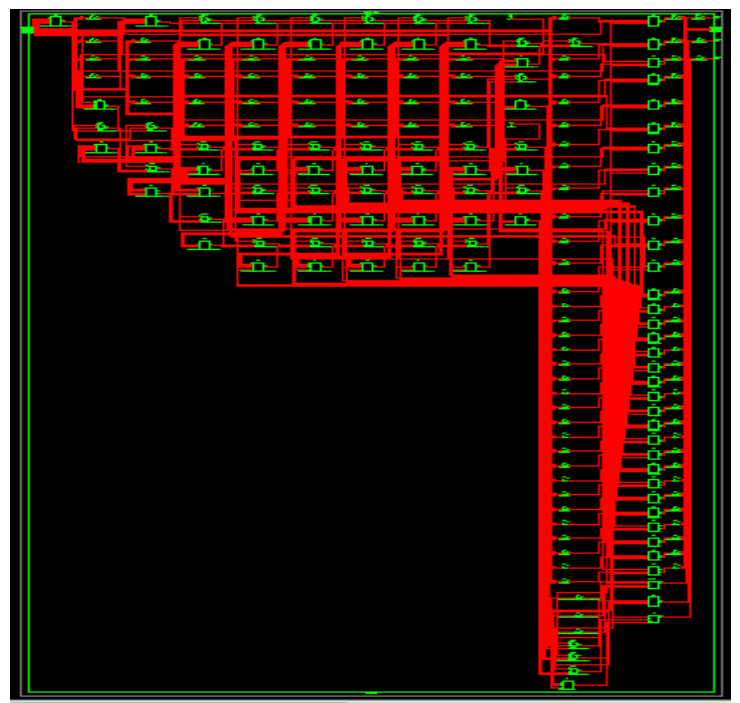

Fig. 5. Detailed view of the business model battery pack.

\section{RESUlTS AND DisCUSSION}

This part concerns the visualization of simulation results.

\section{A. First Case: $U \_B a t t e r i e<$ Vmin}

The load is supplied by GE as shown in Fig. 6.

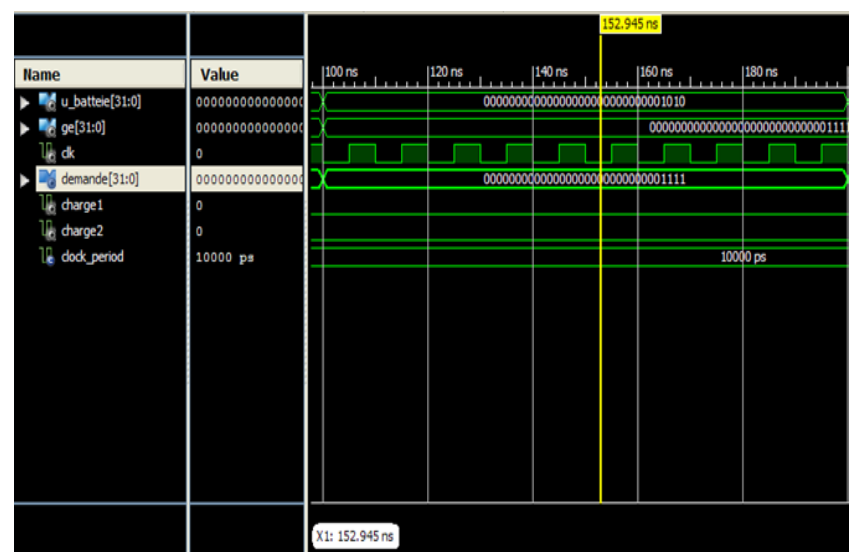

Fig. 6. The load is supplied by GE. 
V1: Supply will be provided by another source (generator GE). The battery units "U_Batterie" which are discharged cannot meet consumer demand because it is below the minimum limit load (Vmin) for this purpose the two charges: load 1 and load 2 are disabled (off).

\section{B. Second Case: Vmin $<U_{-}$Batterie $<$Vmax}

The load is supplied by the battery as shown on Fig. 7 .

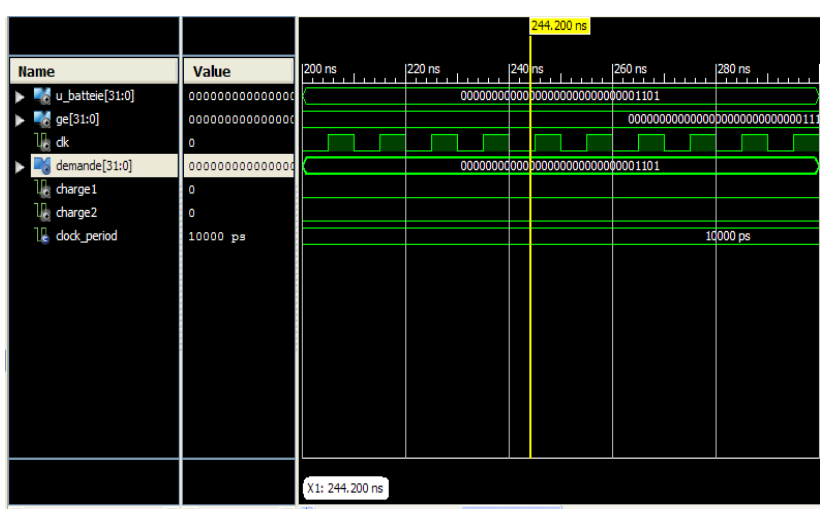

Fig. 7. The load is supplied by the battery

V2: Energy consumption will be provided by "U_Batterie" which is between starting Vmin and the upper limit. GE load 1 and load 2 are deactivated (off).

\section{Third case: $U \_B a t t e r i e>V \max$}

The load 1 is powered by the battery as show on Fig. 8 .

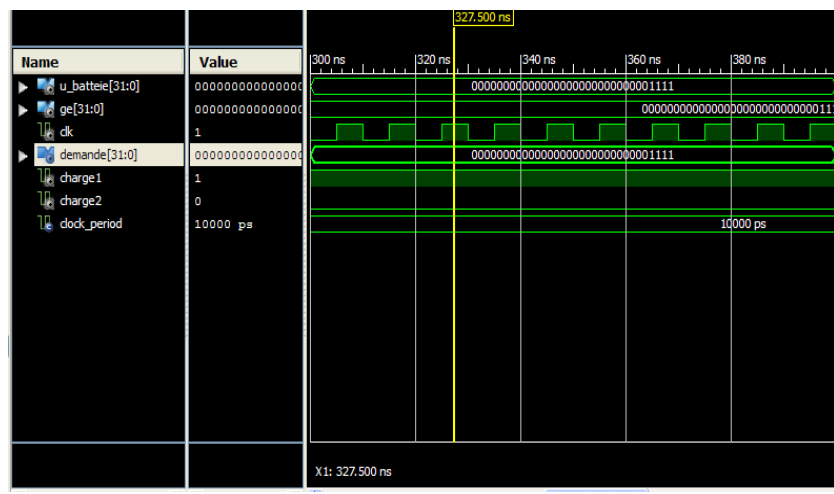

Fig. 8 . The load 1 is powered by the battery.

V3: "U_Batterie" exceeds Vmax, power will always supplied by "U_Batterie" but trigger only a single load "load1" and deactivate load 2 and "GE".

\section{Last Event: U_Batterie $>2$ Vmax}

Both load 1 and 2 are powered by the battery as shown on Fig. 9.

V4: in this case, U_Batterie exceeds twice Vmax, and V3, but we must activate "load 2".

According to the scheme and correspondence with the four stages mentioned above, the battery model works as follows:

V1: Supply will be provided by GE and load 1 and load 2deactivated (off).

V2: Supply will be provided by "U_Batterie", GE, load 1 and load 2 are deactivated (off).

V3: Supply will be provided by "U_Batterie", only load 1 activated; GE and load 2 are deactivated (off).

V4: Supply will be provided by "U_Batterie", load 1 and load 2 are activated and GE deactivated.

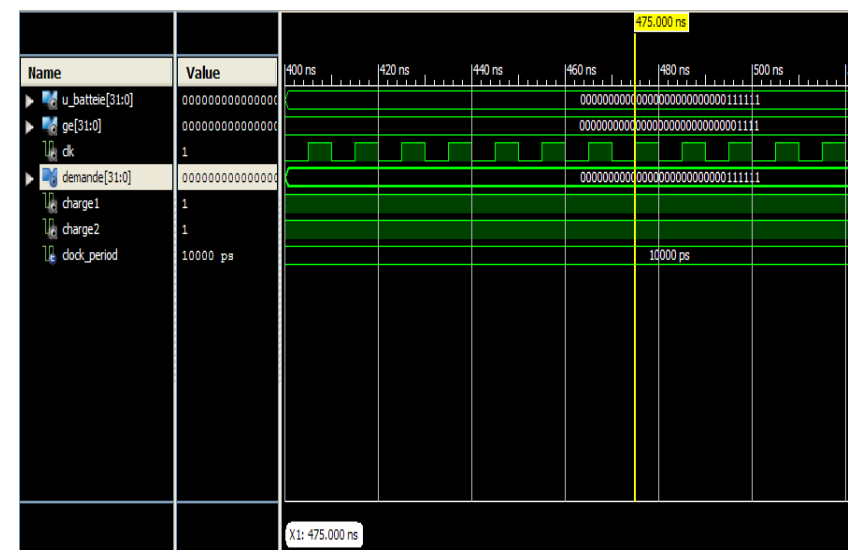

Fig. 9. The load 1 and 2 are powered by the battery.

From Fig. 10 the application is powered by GE during $100 \mathrm{~ns}$ because U_Batterie $<\mathrm{Vmin}$. After this period, the batteries will be charged and the power to the load is switched to the batteries Vmin $<$ U_Batterie $<$ Vmax.

If there is a low demand, the surplus energy will be consumed by the loads (01 and 02 ) which are pumps or engines used for auxiliary services. We must keep the battery charge between the limits $V \min <\mathrm{U} \_$Batterie $<\mathrm{Vmax}$.

In this case, the battery plays a role of an energy regulator, when other sources (PV, wind) do not produce sufficient electricity.

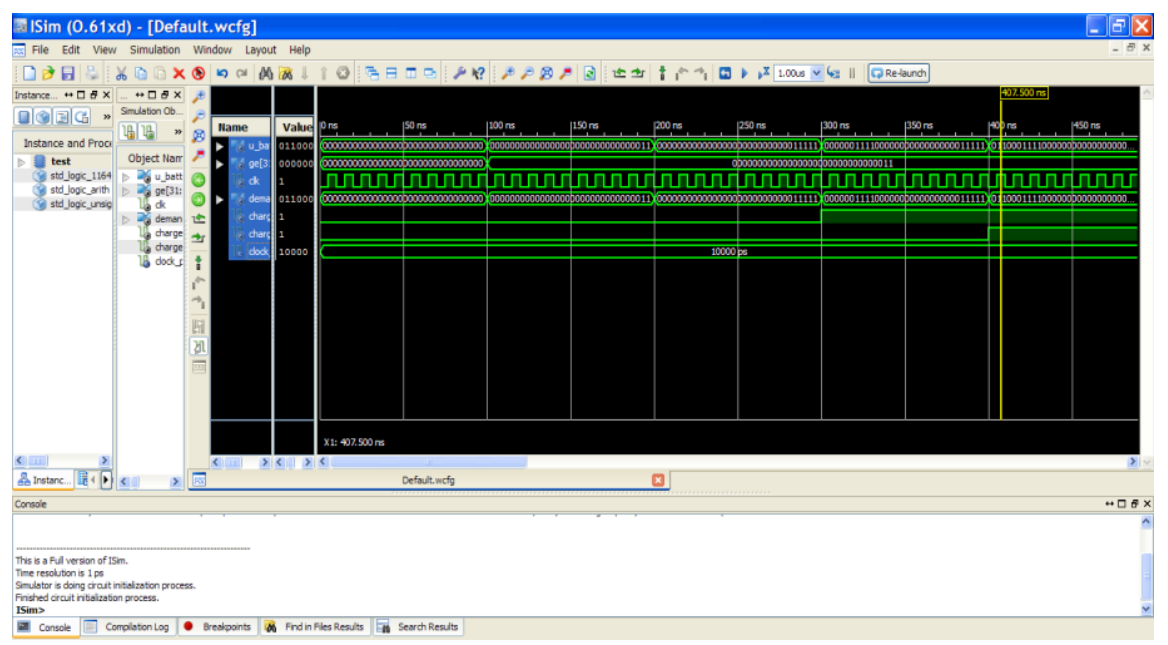

Fig. 10. Global view of the timing of battery management unit. 


\section{CONCLUSION}

The integration of FPGA systems in the management of energy sources is used to monitor the operation of the system to study and obtain a simulation model that can validate a solution before making the component.

Based on these simulations, we proposed a VHDL design and implementation of different applications for multisource systems.

The design and integration of FPGA elements ensuring dependability to a control system was presented.

The proposed model simulation can be changed depending on the input / output of each system. So, it is an advantage of linking several blocks of such systems in order to have a combination of a new model that optimizes energy management of the full installation.

Our purpose is to have an optimized management model of a hybrid system, obtained by a combination of several sub-models of each source controlled separately.

\section{REFERENCES}

[1] A. H. Maghrabi, "Parameterization of a simple model to estimate monthly global solar radiation based on meteorological variables, and evaluation of existing solar radiation models for Tabouk, Saudi Arabia," Energy Conversion and Management, 2009.

[2] B. S. Borrowy and Z. M. Salameh, "Methodology for optimally sizing the combination battery bank and PV array in a wind/PV hybrid system," IEEE Transaction on Energy Conversion, vol. 12, no. 1, pp. 73-78, March 1997.

[3] A. N. Celik, "Optimisation and techno-economic analysis of autonomous photovoltaic-wind hybrid energy system," Energy Conversion and Management, vol. 43, pp. 2453-2468, 2002.

[4] C. T. Ibrahim, "Contribution to the development of models for the power electronics in VHDL-AMS," PhD thesis, National Institute of Applied Sciences OF Lyon, France, 2009.

[5] D. L. Perry, VHDL: Programming by Example, The McGraw-Hill Companies, United States of America, 2002.

[6] E. O. Hwang, Digital Logic and Microprocessor Design With VHDL, Educational Courses, National Polytechnic Institute of Grenoble, 2005.

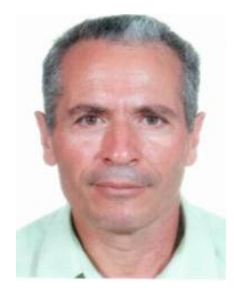

Rezak Alkama was born in Bejaia, Algeria on 1959 $\mathrm{He}$ received his $2^{\text {nd }}$ degree in physics from Setif University, Algeria in 1982. He received his doctorate degree in electrical engineering from Paris VI University, France in 1986. He is a teacher and researcher at Béjaia University, Algeria and responsible of the team "Energy and Environment" in the Electrical Engineering Laboratory of Béjaia. Alkama has many publications in his main subjects of interests as instrumentation, renewable energy and environment. 